\title{
Digital Beamforming Architecture and Techniques for a Spaceborne Interferometric Ka-Band Mission
}

\author{
Marwan Younis, Paco López-Dekker, Anton Patyuchenko, and Gerhard Krieger \\ German Aerospace Center (DLR), Microwaves and Radar Institute \\ Oberpfaffenhofen, Germany \\ Email: marwan.younis@dlr.de
}

\begin{abstract}
The paper presents the instrument concept and performance of a Ka-band single-pass interferemetric mission proposed for measuring topography and topographic changes. A formation flying constellation of two compact synthetic aperture radars (SAR) satellites equipped with innovative digital beamforming hardware and advanced operation modes is described.
\end{abstract}

\section{INTRODUCTION}

Recently, a team of scientist lead by the German Aerospace Center (DLR) have proposed a Ka-band single-pass interferometric mission concept named SIGNAL (SAR for Ice, Glacier aNd globAL Dynamics) [1]. The main goal of SIGNAL is to estimate accurately and repeatedly topography and topographic changes variations associated with mass change or other dynamic effects on glaciers, ice caps and polar ice sheets. To achieve the required elevation accuracy, in the order of a few decimeter, a formation flying constellation of two compact satellites was proposed as the only way to obtain the desired cross-track baseline.

This paper presents a new evolution of SIGNAL's instrument concept that uses novel Digital Beamforming (DBF) techniques to further extend the coverage of the mission. In addition, by introducing two phase centers in azimuth, which are needed to achieve the swath extension, the system gains along-track interferometric capabilities. These may be used, for example, to measure ocean currents. Besides these wide-swath and ATI-modes, the paper will also discuss pingpong multi-baseline acquisitions modes exploiting a novel subpulses technique in order to keep a low PRF.

\section{System ARChitecture}

As in most studies for Ka-band SAR missions, a reflector based architecture was chosen. Here, however a digital feed array is proposed, such an architecture has the potential to combine both the flexibility and the capabilities of digital beamforming with the high antenna gain provided by a large reflector aperture. The idea of applying digital beamforming to a reflector system was first suggested in [2].

This section first describes the DBF techniques utilized by the SAR instrument and its architecture. A salient feature of the architecture proposed in the unconventional use of of two transmit antennas with a single receive one. Most of the mutual interference problems associated to this choice are overcome by the introduction of a novel sub-pulses technique, which is discussed in the following.

\section{A. Antenna Configuration and Instrument Architecture}

Unlike conventional approaches the antenna configuration here uses two transmit antennas separated in flight direction as shown in Fig. 1. This provides two separate phase centers (samples) which can be used either to improve resolution or for ATI applications. Each Tx reflector is $3 \times 0.6 \mathrm{~m}$ (azimuth $\mathrm{x}$ elevation) and has a $2 \mathrm{~m}$ focal length. The Tx reflectors are placed at the end of $2 \mathrm{~m}$ booms which are unfolded during deployment in orbit. The reflectors are illuminated by a feed arrays mounted on the satellite body ( $2 \mathrm{~m}$ offset), which avoids laying waveguides and active RF circuitry on the deployable boom. The wide beam of the two transmit reflectors illuminate the same area on the ground, the half power beamwidth is set to $2.9 \times 0.16^{\circ}$ in elevation and azimuth, respectively.

Each transmit antenna has its RF power supply consisting of an extended interactive klystron (EIK). A peak power of $\approx 6 \mathrm{~kW}$, depending on the required bandwidth, is state-ofthe-art performance. Note that the distributed power approach using two EIKs enable a doubling of the available RF transmit power, without using power combiners.

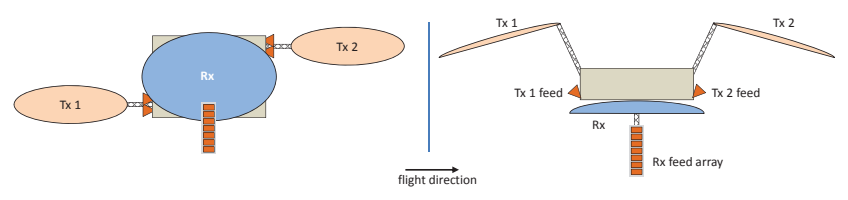

Fig. 1. Antenna configuration with the two transmit (Tx) reflectors placed at the end of the booms, while the receive $(\mathrm{Rx})$ reflector is fixed to the platform.

A single reflector antenna is used on-receive. The size of the dish antenna is $3 \times 2 \mathrm{~m}$ which is larger (in elevation) than the transmit reflectors. The reflector is fix mounted on the satellite platform and illuminated by a $0.4 \mathrm{~m}$ feed array arranged in the plane perpendicular to the flight direction and mounted on a deployable boom in an offset configuration. The digital feed array of 27 receive elements is used for SCORE operation (see section II-B) [2]. Each element results in a narrow beam, illuminating a region on the ground, which partially overlaps with the region illuminated by the beams of the adjacent elements. To illuminate a given angular segment in elevation the corresponding feed elements are activated. Here each set of elements is only active during a subinterval of this time period. The signals of the feed elements are combined on-board using time variant complex coefficients to form the SCORE beam. 
The receive beam will scan the complete swath within the time period of one $P R I$.

The $\mathrm{Rx}$ antenna system is equipped with additional scanning capabilities such that the SCORE beams span an angular segment of $\pm 2.9^{\circ}$. This way the Rx antenna can cover double the swath of the Tx antenna. Such a configuration allows doubling the total swath in bistatic operation mode as will be explained later. This also means that the two satellite designs are not identical, since the the Tx illumination overlaps with the upper part of the Rx pattern for one satellite and with the lower part for the other.

\section{B. Digital Beamforming Techniques}

The SCan-On-Receive (SCORE) mode of operation is primarily based on generating a wide transmit beam and a narrow, high gain beam on receive that follows the pulse echo on the ground [3], [4]. The high gain SCORE beam results in an increased signal-to-noise ratio, compensating the low gain (wide beam) transmit antenna loss. Further, the narrow receive beam has the advantage of attenuating the range ambiguities. Since the direction of arrival of the received echo is a function of the slant range, a one-to-one relation exists between the required beam steering angle and the time variable. DBF is used to obtain a beam that chases the echo while it traverses the ground. The specific beamforming implementation depends on the system involved, but can in general be described by a complex and time varying weighting of the antenna feed signals followed by a summation (data reduction).

As known, the use of multiple phase centres in flight direction eliminated the tight coupling between azimuth resolution and unambiguous swath-width applicable to a single channel system. Alternatively, these multiple phase centers enable along track interferometry (ATI). In this paper we propose a sub-pulse illumination technique; the basic idea is to transmit two sub-pulses, one from each Tx antenna within one $P R I$ period (c.f. Fig. 2) which illuminate the same swath on the ground. Considering a single Tx antenna (and disregarding the ATI case for a moment) the spatial separation between the samples, as determined by the pulse repetition frequency $(P R F)$, is such that the Doppler spectrum would be undersampled, i.e. aliased or ambiguous. It is only through the combination of the spatial samples of all Tx channels, that the Doppler spectrum is recovered unambiguously. Further, in most cases the system $P R F$ will result in a non-uniform spatial sampling; this makes the combination of the channels a non-trivial task, requiring a reconstruction of the Doppler spectrum as detailed in [5] which based on a generalization of the sampling theorem [6].

The challenge is to separate the signals from the two subpulses which are transmitted consecutively illuminating the same swath which causes overlapping echo windows. The subpulses can be thought of as range ambiguous with respect to each other. The "trick" here is to utilize the fact that at each time instance the two echoes are arriving from slightly different (time varying) angles and can thus be separated by the directive SCORE beams and digital beamforming as

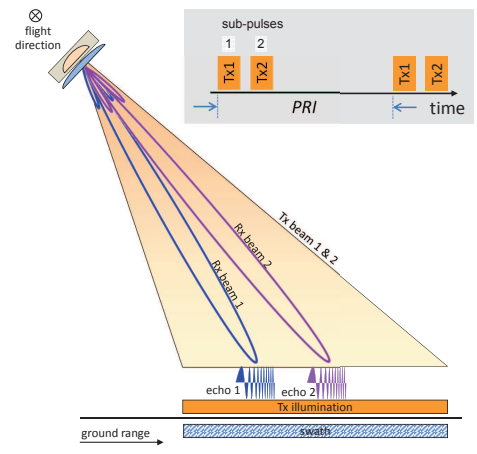

Fig. 2. Single Satellite Stripmap operating mode where two sub-pulses are used to illuminate the same swath which are separated by the two SCORE beams.

indicated in Fig. 2. This angular sub-pulse separation does not require any additional hardware w.r.t. a conventional singlebeam SCORE system.

\section{Operation MODE}

Each instrument is designed for single pass along-track interferometry, or SAR imaging with two azimuth phase centres. Using two satellites allows various operation modes with one or more combinations of ATI, XTI, wide swath and high resolution. In the following a selection of these modes are discussed (the sub-pulse technique is utilized in all modes).

\section{Single Satellite Stripmap}

The basic operation uses one satellite operating in the stripmap mode (c.f. Fig. 2). Here the sub-pulse technique with the two Tx antennas allow for two azimuth samples per $P R I$. This can be understood as enabling a reduced $P R F$ and thus an increased swath, or equivalently an improved azimuth resolution despite the low $P R F$. In any case a sample reordering (as detailed for example in [7]) is necessary, since the two Tx antennas are non-contiguous.

\section{Single Satellite ATI}

For sufficiently high $P R F$, an along-track interferometric operation mode is possible. This is facilitated through the two Tx antennas, where the PRF needs to be high enough to result samples from different pulses being at the same azimuth position. In this mode the swath width is reduced due to the timing constraints caused by the higher PRF and the along-track interferometric operation is provided at the cost of worsening the azimuth resolution. Due to the fixed beam Tx antenna, the swath illuminated is larger than the imaged strip. This may allow imaging different incident angles only by changing the $P R F$.

\section{Two Satellite bistatic XTI}

Here as shown in Fig. 3 an across-track operation is feasible. The two satellite simultaneously illuminate two swathes separated by a blind gap. Due to the extended scan capabilities of the receive antenna, each $\mathrm{Rx}$ antenna receives the echoes from the two swathes. In total, each satellite has 4 SCORE 
beams to separate the time overlapping echoes. This mode thus produces a total of two monostatic and two bistatic images corresponding to two swathes. The XTI imaged swath is thus doubled.

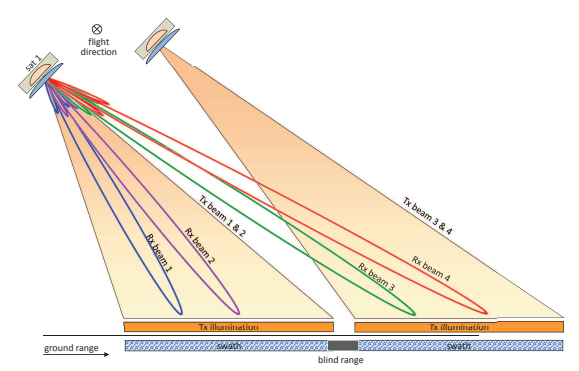

Fig. 3. Two satellite cross-track interferometric operation mode (Rx beams of satellite 2 not shown).

\section{Two Satellite combined XTI and ATI}

The only difference between this mode and the previous one is that the $P R F$ is increased to create overlapping azimuth samples. Again the timing will limit the swath width but on the other hand provide simultaneous XTI and ATI imaging. As before, different swathes may be covered or, alternatively, the same swath may be observed under distinct squint angles in order to obtain a 2-D ATI measurement.

\section{SAR PERFORMANCE}

In the following the main SAR performance of the system operating at $35.75 \mathrm{GHz}$ are reported. The mode shown here is for the single satellite stripmap case, since this can be regarded as a reference for the other modes.

\section{A. Timing and swathes}

The satellite operates in a $740 \mathrm{~km}$ orbit. Different swathes can be imaged by tilting the satellite to point at the respective swath. Here the performance is shown for the near range swath at an incidence angle of $34-37^{\circ}$ which corresponds to $50 \mathrm{~km}$ swath width.

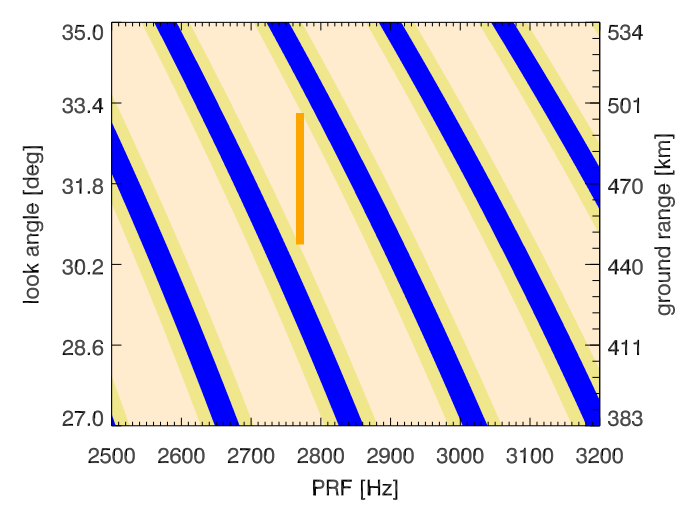

Fig. 4. Timing diagram for a $740 \mathrm{~km}$ orbit and the near-range swath of $50 \mathrm{~km}$ swath.

The combined Tx reflector lengths of $6 \mathrm{~m}$ set the minimum required $P R F$. One particularity of reflector patterns that has to be kept in mind is that the azimuth and elevation patterns are not separable. This point is crucial both for the system design and the performance computation; it means that the azimuth pattern shape will depend on the elevation scan angle, i.e. the azimuth patterns degrade at the swath edges. For the system considered here the PRF has to be increased to $2770 \mathrm{~Hz}$ to compensate for the broader azimuth patterns. The subpulse duty cycle is $10 \%$ which together with the sub-pulse separation and the $P R F$ determines the available echo window length.

\section{B. Range-Ambiguity-to-Signal Ratio}

Reflector based SAR systems have very low rangeambiguity level, because of the low sidelobe level of the Tx beam and the narrow Rx beam. When using sub-pulses the range ambiguities have to be carefully considered, because the angular separation between the sub-pulses is determined by their temporal separation during transmission. Further, the transmit patterns will not suppress sub-pulses since the same swath is illuminated. This is seen in Fig. 5(a) which shows an instantaneous elevation pattern with the ambiguity and signal position marked. The $R A S R$ performance is shown in Fig. 5(b), the level is better than $-20 \mathrm{~dB}$. However, this level is achieved optimizing the weighting coefficients of the $\mathrm{Rx}$ patters [8]. Here the time separation between sub-pulses is adapted depending on the imaged swath so that the required ambiguity performance is achieved.

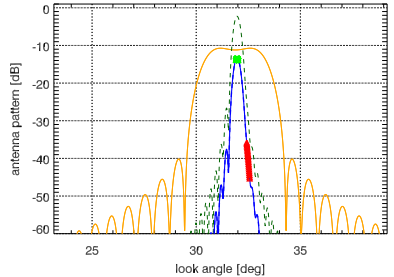

(a) Elevation pattern

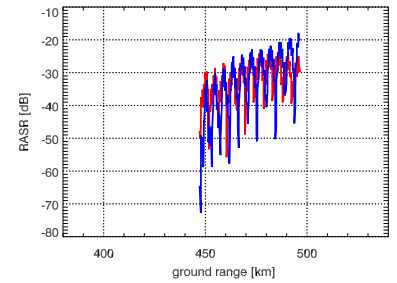

(b) $R A S R$
Fig. 5. An example of an elevation patter showing the position of the signal and ambiguous sub-pulse energies (left) and the resulting range-ambiguityto-signal ratio.

\section{Azimuth-Ambiguity-to-Signal Ratio}

Fig. 6 shows an example azimuth pattern at one elevation cut and the $A A S R$ after azimuth reconstruction. The processed Doppler bandwidth corresponds to an azimuth resolution of $6.8 \mathrm{~m}$.

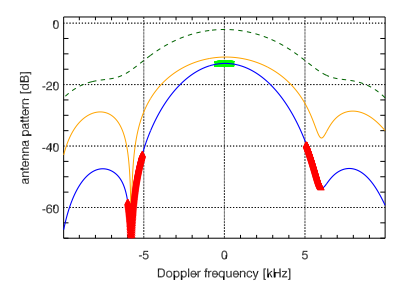

(a) Near range azimuth pattern

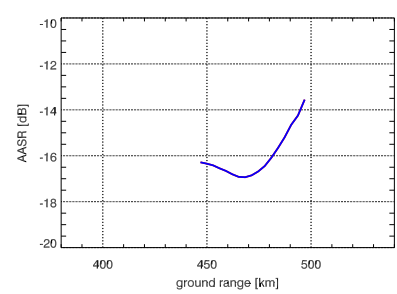

(b) $A A S R$
Fig. 6. Antenna patterns and azimuth ambiguity-to-signal ratio (AASR). 
As expected the $A A S R$ shows a variation over the swath, which is understood from the above discussion and the azimuth patterns in Fig. 6(a); the position of the signal and ambiguities is indicated by the green and red lines on the pattern, respectively. Even though poor, the $A A S R$ performance is achieved by azimuth oversampling, i.e. high $P R F$, which comes at the expense of a higher data rate.

\section{Noise-Equivalent Sigma-Zero}

Although this performance parameter profits from the reflector gain, but still other instrument and operation parameters influence its value. For example a small number of feed array elements would be activated to achieve a high receive antenna gain; however this would cause a narrow receive beam which -depending on the pulse duty cycle- might not cover the complete ground extension of the pulse.

The radiometric performance of the system is shown in Fig. 7(a) in terms of the noise-equivalent sigma zero (NESZ) for an average power of $600 \mathrm{~kW}$ per transmitter. chirp bandwidth of $40 \mathrm{MHz}$. Note that NESZ requirements at Ka-band is somehow relaxed because of the higher ground reflectivity.

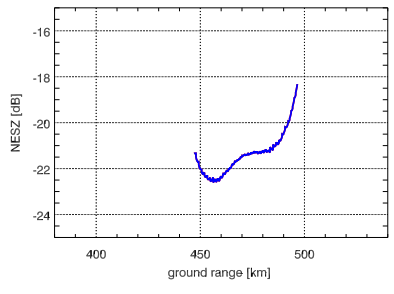

(a) $N E S Z$

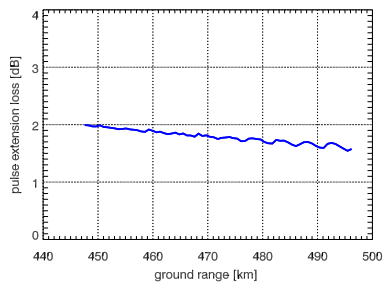

(b) $P E L$
Fig. 7. SNR performance of the system in term so the noise-equivalent sigma-zero and the pulse extension loss.

Fig. 7(b) shows the pulse extension loss (PEL) which indicates the loss due to the pulse attenuation through the narrow SCORE receive beam [9] and has a direct impact on the $S N R$. The $P E L \approx 2 \mathrm{~dB}$ can be reduced by using a frequency selective filter in the SCORE processing as detailed in [8].

\section{LEVEL-2 PERformanCE}

This section briefly discusses the performance at Level-2 product level.

\section{A. DEM errors}

The main product of SIGNAL are high precision DEMs, whose performance is given in terms of the $90 \%$ point-to-point relative error $\left(\Delta h_{e r r}^{90 \%}\right)$. For a discussion of how these errors are estimated for SIGNAL the reader is referred to [10].

As discussed in previous papers, the SIGNAL mission concept includes a DEM acquisition phase, where larger height of ambiguities would be used in order to avoid phase unwrapping problems, and a DEM tracking phase with $h_{a m b}$ in the order of $10 \mathrm{~m}$. Figure 8(b) shows the expected value for $\Delta h_{e r r}^{90 \%}$ during this DEM tracking phase for several surface types assuming a $100 \times 100 \mathrm{~m}^{2}$ product resolution. For the performance computation, $2 \mathrm{~dB}$ of atmospheric attenuation were included.
For dry-snow covered surfaces, bare soil, and even grass, $\Delta h_{e r r}^{90 \%}$ is between 10 and $15 \mathrm{~cm}$, which is comparable to what can be achieved with nadir-looking altimeters. Several factors are key to this very good performance:

- High coherence values mainly due to the avoidance of temporal decorrelation. Note that the coherence is mainly SNR driven.

- The high number of looks associated to the relaxed product resolution. It is worth noting that even at $12 \mathrm{x}$ $12 \mathrm{~m}^{2}$ resolution, the error stays below the $2 \mathrm{~m}$ threshold given corresponding to the HRTI-3 standard.

- The possibility to use large baselines, without which it becomes practically impossible to achieve these level of performances over relatively wide swaths.

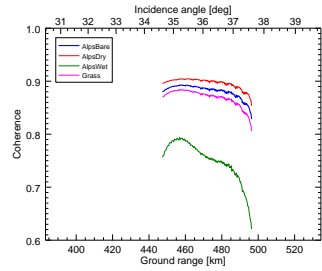

(a) $\gamma$

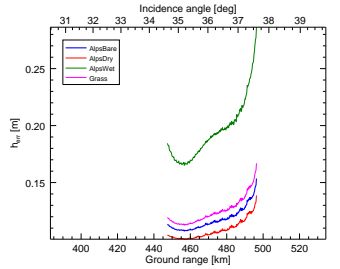

(b) $\Delta h_{e r r}^{90 \%}$
Fig. 8. XTI performance for a $100 \times 100 \mathrm{~m}^{2}$ resolution product and 10 $\mathrm{m}$ height of ambiguity. Left: interferometric coherence. Right: $90 \%$ point-topoint relative error $\left(\Delta h_{e r r}^{90 \%}\right)$.

\section{B. Surface velocity error}

The new proposed architecture allows ATI measurements, which can be particularly useful for the measurement of ocean currents. In principle, since the design considers a single azimuth beam, only one component of the surface velocity can be measured. However, applying an opposite yaw rotation to both spacecraft allows observing the same ocean surface with two different beams, thereby implementing a distributed dualbeam interferometer (DBI). For the preliminary performance calculations shown in Figure 9, a $45^{\circ}$ rotation has been assumed so that the two measured surface components are, in theory, mutually orthogonal. Since the expected performance is better for steeper incident angles, a 15 degree roll rotation has also been assumed. Last, a 10 meter physical baseline between the two Tx phase centers has been considered. Note that this baseline is mainly constrained by mechanical limitations.

Figure 9 shows the predicted standard deviation of the error of the magnitude of the estimate surface current as a function of the wind direction relative to broad-side (where $0^{\circ}$ means that the radar looks into the wind) and of range. A product resolution of $1 \mathrm{~km}^{2}$ has been assumed and $10 \mathrm{~m} / \mathrm{s}$ wind conditions have been assumed. For details on the performance calculations the reader is referred to [11]. This preliminary results are encouraging in that the typically desired error level of $10 \mathrm{~cm} / \mathrm{s}$ is reached across the swath for some conditions. Nevertheless, it is clear that the performance must be improved in order to satisfy typical user requirements under a wider range of conditions. 


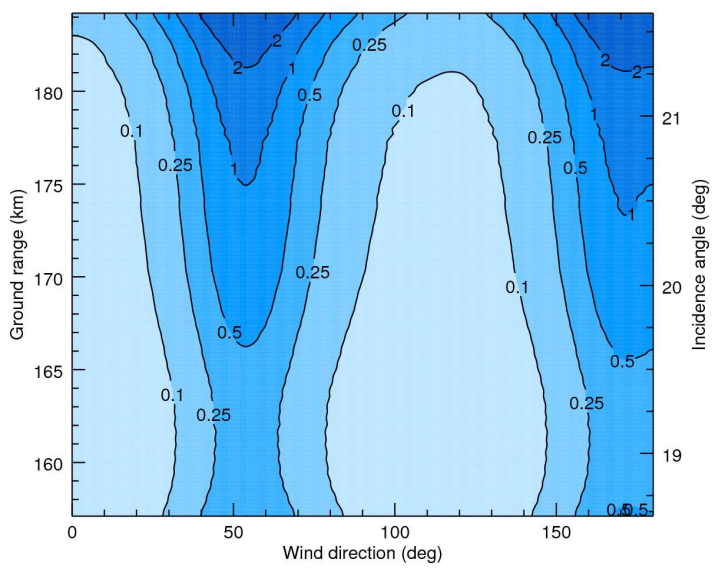

Fig. 9. Standard deviation of the error of the magnitude of the estimated surface current. A product resolution of $1 \mathrm{~km}$ is assumed for this plot.

An attractive feature of the distributed DBI concept, is that if the two spacecraft are flown with a sufficiently large alongtrack separation, a third bistatic beam may be formed. This third beam could be used to mitigate biases in the Doppler velocity measurements.

\section{SUMMARY}

The paper presents a concept for a spaceborne interferometric Ka-band SAR that uses digital beamforming in conjunction with novel sub-pulse illumination techniques. The performance results shown represent the current preliminary status of the investigation where the design parameters can further be optimized. Nevertheless, the system concept presented is considered highly attractive for future Ka-band SAR missions.

Future work will try to optimize the system and mission concept in order to improve the ocean current measurement performance. A key ingredient for this optimization may be lowering the orbit from the current $740 \mathrm{~km}$ to something in the order of $500 \mathrm{~km}$. A second ingredient my be increasing the effective ATI baseline, be it by increasing the separation between the transmitters, or by considering using also two receivers.

\section{REFERENCES}

[1] T. Börner, F. De Zan, P. Lopez-Dekker, G. Krieger, I. Hajnsek, K. Papathanassiou, M. Villano, M. Younis, A. Danklmayer, W. Dierking, T. Nagler, H. Rott, S. Lehner, T. Fugen, and A. Moreira, "SIGNAL: SAR for ice, glacier and global dynamics," in Geoscience and Remote Sensing Symposium (IGARSS), 2010 IEEE International, July 2010, pp. 2884-2887.

[2] G. Krieger, N. Gebert, M. Younis, and A. Moreira, "Advanced synthetic aperture radar based on digital beamforming and waveform diversity," in Proc. IEEE Radar Conference (RadarCon), Rom, Italy, May 2008.

[3] M. Suess and W. Wiesbeck, "Side-looking synthetic aperture radar system," European Patent Patent EP 1241 487, Sept., 2002.

[4] G. Krieger, N. Gebert, M. Younis, F. Bordoni, A. Patyuchenko, and A. Moreira, "Advanced concepts for ultra-wide swath SAR imaging," in Proc. European Conference on Synthetic Aperture Radar EUSAR'08, Friedrichshafen, Germany, June 2008.
[5] G. Krieger, N. Gebert, and A. Moreira, "Unambiguous SAR signal reconstruction from non-uniform displaced phase centre sampling," IEEE Geoscience and Remote Sensing Letters, vol. 1, no. 4, pp. 260264, Oct. 2004

[6] A. Papoulis, "Generalized sampling expansion," IEEE Transactions on Circuits and Systems, vol. 24, no. 11, pp. 652-654, Nov. 1977.

[7] C. Schaefer, M. Völker, P. López-Dekker, M. Younis, E. DaganzoEusebio, and M. Ludwig, "Space-borne ka-band across-track SAR interferometer," in Proc. 1st workshop on ka-band earth observation radar missions (KEO’12), Nov. 2012.

[8] S. Huber, M. Younis, G. Krieger, A. Patyuchenko, and A. Moreira, "Spaceborne reflector SAR systems with digital beamforming," IEEE Aerospace and Electronics Systems Magazine, accepted, to be published in 2012.

[9] M. Younis, S. Huber, A. Patyuchenko, F. Bordoni, and G. Krieger, "Performance comparison of reflector- and planarantenna based digital beam-forming SAR," Int. Journal of Antennas and Propagation, vol. 2009, June 2009. [Online]. Available: http://www.hindawi.com/journals/ijap/2009

[10] P. López-Dekker, T. Börner, M. Younis, and G. Krieger, "SIGNAL: A Ka-band digital beam-forming SAR system concept to monitor topography variations of ice caps and glaciers," in Proceedings of Advanced RF Sensors and Remote Sensing Instruments (ARSI), Sept. 2011, pp. 1-10. [Online]. Available: http://elib.dlr.de/72745/

[11] S. Bertl, P. López-Dekker, S. Baumgartner, J. G. Molina, M. Younis, F. Bordoni, C. Tienda-Herrero, C. Schaefer, and G. Krieger, "Ska-band multi-baseline ati-sar system for ocean surface currents measurements," in Proc. 1st workshop on ka-band earth observation radar missions (KEO?12), Nov. 2010. 